\title{
Lysosomes in cancer cells
}

\author{
A. C. Allison \\ From the Clinical Research Centre, Harrow
}

Lysosomes are membrane-bounded cytoplasmic organelles containing hydrolytic enzymes. They play a major role in intracellular digestion in a wide range of animal cells from amoebae to mammalian phagocytes. Two processes by which materials are taken into cells before digestion can be distinguished: phagocytosis of particles and pinocytosis of nonparticulate macromolecules. In both cases endocytic vacuoles are formed which fuse with lysosomes containing hydrolytic enzymes. The macromolecules are digested and the low molecular weight products (dipeptides, monosaccharides, and others) pass through the lysosomal membrane into the cytoplasm. When cells die as a result of inadequate blood supply or for other reasons, enzymes are released from lysosomes and are mainly responsible for aseptic autolysis.

Lysosomes are also involved in other cellular changes. Under certain conditions organelles which are normally separate, eg, mitochondria, rough- and smooth-membrane endoplasmic reticulum, and lysosomes, become enclosed in smooth-membrane organelles known as autophagosomes. Digestion of the constituents of autophagosomes can occur without loss of cell viability. Surviving cells can also under certain conditions secrete lysosomal hydrolases into the extracellular environment, and the hydrolases can break down connective tissue proteoglycan matrix as well as collagen fibres. The use of antibodies against purified enzymes is now helping to define their role in these processes. Proteases can also cleave the carbohydrate moieties from membrane glycoproteins, release cells from density-dependent inhibition of mitosis, and liberate cells from aggregates.

Many of these processes are of interest in tumour cells. Although they are seldom phagocytic, tumour cells often exhibit considerable pinocytic capacity. This may play a role in the nutrition of tumour cells and could provide a means for achieving a selective concentration of chemotherapeutic agents in these as contrasted with normal host cells. Autolysis often occurs in tumours, and liberation of lysosomal hydrolases could contribute to inflammatory and toxic effects. Autophagy appears to be a major cause of regression when tumours are deprived of the hormones on which growth is dependent, and is also seen when tumours are treated by chemotherapy or radiotherapy. It is likely that hydrolase release from tumour cells is required for digestion of connective tissue matrix and fibrils in basement membranes and other sites during invasion by malignant cells. Released lysosomal hydrolases may promote detachment of cells from tumour masses and so facilitate metastatic spread. In view of the effects of hydrolases on density-dependent inhibition, they may even play some part in the distinction between normal and malignant cells. In this paper available information on the role of lysosomes in tumour cells is outlined.

\section{Lysosomal Enzymes in Tumour Cells}

As recently reviewed by Poole (1973), several studies have revealed higher specific activities of lysosomal enzymes in solid tumours than in their tissues of origin. These include beta-glucuronidase in tumours of the mouse (Karunairatnam, Kerr, and Levvy, 1949; Cohen and Bittner, 1951) and humans (Fishman and Anlyan, 1947; Boyland, Wallace, and Williams, 1955;Watts and Goldberg, 1969), and beta$\mathrm{N}$-acetylglucosaminidase (Conchie and Levvy, 1957) and arylsulphatases in human tumours (Boyland et al, 1955; Dzialoszynski, Fröhlich, and Kroll, 1966). Shamberger and Rudolph (1967) have found increased acid phosphatase, cathepsin $\mathrm{D}$, and betaglucuronidase in mouse skin cancers as compared with normal skin. Goldberg and Pitts (1966) found higher activities of acid ribonuclease and deoxyribonuclease in the cytoplasmic fractions prepared from human cancers of the cervix as compared with norrihal cervical tissue, and Deckers-Passau, Maisin, and de Duve (1957) found more cathepsin $D$ and acid nucleases in hepatoma than in normal rat liver. Fibroblasts transformed by viruses had much higher activities of seven glycosidases (per cell or per unit protein) than the corresponding untransformed cells (Bosmann, 1969).

There is also evidence that invasiveness of tumours shows a relationship to their content of lysosomal 
enzymes. Increased invasiveness and a high mitotic index of tumours of the female genital tract is correlated with levels of cathepsin B1 (Blackwood, Mandl, and Long, 1965) and beta-glucuronidase (Watts and Goldberg, 1969). Transplantable tumours grown subcutaneously showed higher activities of beta- $\mathrm{N}$-acetylglucosaminidase and beta-glucuronidase than when grown intraperitoneally in a mainly non-invasive form.

Several factors must be taken into consideration when discussing these results. One is that cornifying cells in squamous epithelia, such as those of skin and cervix, have low lysosomal hydrolase contents. It is not surprising that tumours of these organs should have greater lysosomal enzyme activities than the corresponding normal tissues. The second point is that rapidly growing normal tissues show greater lysosomal enzyme activities than their normal counterparts. Thus, regenerating liver has higher acid phosphatase activity than normal liver (Adams, 1963), and newborn liver and kidney have higher activities of this enzyme (Franklin, 1962) than the adult tissues. The same is true of arylsulphatases A and B in newborn as compared with adult liver (Roy, 1958) and for beta-glucuronidase in a variety of rapidly growing tissues (Levvy, 1956; Watanabe and Fishman, 1964). This does not account for differences between solid and ascitic tumours which grow at approximately the same rates.

A third factor is pinocytosis, which, as discussed below, is well marked in tumour cells. In macrophages (Cohn and Fedorko, 1969) stimulation of pinocytosis leads to considerable elevations in lysosomal hydrolase levels. Possibly cell-to-cell contact in tumours also helps to maintain relatively high lysosomal enzyme levels, as the differences between the same tumour in solid and ascitic form suggest. When secretion of hydrolases into the extracellular medium is stimulated in cartilage cells (Dingle, 1969) and macrophages (Page, Davies, and Allison, 1972), considerable synthesis of the enzymes takes place, and it is conceivable that the enzyme activities of tumours partly follow from overcompensation of the loss from this cause.

There are also technical problems when analysing the total activities and distribution of hydrolases in subcellular fractions of tumours. Among these are the presence of dead cells, secreted enzymes, and large secondary lysosomes which are readily disrupted by homogenization. All of these may decrease the latency of hydrolases in tumour cells. Although the observed high activities in malignant cells may be correlated to tumour invasiveness it is not yet clear whether there is a cause-and-effect relationship.

\section{Distribution of Enzymes in Tumour Cell Particles}

This subject has also been reviewed by Poole (1973). When cells are carefully homogenized in isotonic medium, so as to avoid disrupting cytoplasmic organelles, high proportions of the hydrolytic enzymes are latent in the lysosomal or large granule fractions and low proportions are found in the supernatant fractions. In dead cells and under certain other conditions the distribution is reversed, so that comparison of the distribution of the enzymes and measurements of latency can provide information about the state of the lysosomes in the tissues. In general tumour cells have distribution patterns for lysosomal enzymes in cell fractions similar to those in the corresponding normal cells, but incubation of lysosomes at pH 5.0 and $37^{\circ} \mathrm{C}$ unmasks latent hydrolases to a lesser extent in tumour cells than in normal cells. This has been found in rapidly growing Novikoff hepatoma cells but not in the more slowly growing Reuber hepatoma (Wagner and Roth, 1967; Wattiaux, Wattiaux de Coninck, van Dijek, Dupal, and Morris, 1970), in the rapidly growing Ehrlich ascites tumour cells (Horvat and Touster, 1967), and in the rapidly growing invasive Guerin T8 epithelioma (Poole, 1973). Whether the altered latency of lysosomal enzymes has any pathophysiological significance is unknown.

Histochemical studies have shown lysosomal enzymes such as acid phosphatase and betaglucuronidase to have the usual cytoplasmic granular localization in most tumour cells, although diffuse staining has been reported. In some instances this is apparently due to autolysis, but in others it may be of more general interest. Thus, the localization of beta-glucuronidase, but not that of acid phosphatase, was found by Poole (1973) to vary according to whether a tumour cell is actively invading normal tissues. Away from the invasion site both enzymes showed a particulate distribution whereas close to the invasion site the distribution of acid phosphatase was particulate whereas that of beta-glucuronidase was diffuse. This was interpreted as due to the presence of beta-glucuronidase in small vesicles of the Golgi and primary lysosomal system rather than the larger digestive secondary lysosomes. In the livers of mice belonging to some strains, but not others, beta-glucuronidase is present in the microsomal fraction (Paigen, 1959), and perhaps there is an analogous adaptive change in tumours. Possibly beta-glucuronidase from small vesicles is secreted into the extracellular compartment. Confirmation of these conclusions by electron microscopy and other methods would be of interest.

There are several reports that in tumours the 
proportion of unsedimentable (cytosol) as compared with particulate enzyme is higher than in normal tissues. For example, this is true of glycosidases in several tumours as compared with normal liver or kidney (Conchie and Hay, 1963) for a variety of hydrolases in hepatomas as compared with normal liver (Deckers-Passau et al, 1957). Even though precautions have been taken to homogenize the tumours carefully under optimum conditions, the lysosomes may be disrupted, especially if they are larger than normal (phagolysosomes or autophagosomes). Most tumours contain necrotic areas, in which enzymes are released from lysosomes. Often they also contain infiltrating leucocytes, with high lysosomal hydrolase activities which may be nonsedimentable. Thus the interpretation of nonparticulate enzyme in tumours is difficult.

This is also true of enzymes in samples of tissue fluids from tumours studied by Sylvén (1968a) and Fiszer-Szafarz and Gullino (1970). High lysosomal hydrolase contents were found in these fluids. The relative contributions of secretion from living tumour cells, from autolysis, or from infiltrating leucocytes may vary from tumour to tumour and in different parts of the same tumour. Samples of tissue fluid from hormone-dependent rat mammary carcinomas, which have a good blood supply and show no leucocytic infiltration, had no demonstrable lysosomal enzyme activities (Gullino and Lanzerotti, 1972).

\section{Esterase in Virus-transformed Cells}

In contrast to the increase in several glycosidases in virus-transformed as compared with untransformed cells in culture (Bosmann, 1969) is the marked decrease in the activity of indoxylacetate esterase that is associated with transformation of human diploid lung cells by SV40 (Bartholomew, Bartholomew, and Rose, 1969). An esterase isoenzyme with cathodal electrophoretic migration, which was a large component in the normal cells, was present in only trace amounts in the transformed cells. A similar change has been found in several other cell strains transformed by SV40 (N. R. Rose, personal communication), but not in some other malignant cells, so that it appears to be associated with SV40 transformation rather than being a general property of malignant cells. This enzyme can be shown to be lysosomal by immunofluorescence, using a specific antibody, by histochemistry, and by cell fractionation. The enzyme is an aliesterase sensitive to DFP, and its physiological function is unknown. On theoretical grounds it might be involved in the establishment of 'functional coupling' in normal cells, and if that is true the change during trans- formation would be of considerable interest. In any case, the enzyme provides a marker for SV40 transformation, and why it should be selectively decreased is unknown. The analogy with specific enzyme changes in host cells accompanying integration of a lysogenic phage is suggestive, but no specific site for integration of the SV40 genome has yet been established.

\section{Lysosomal Enzymes and Tumour Invasiveness}

To invade normal tissues malignant cells must penetrate through connective tissues, including basement membranes, and between host cells. The latter would be facilitated if host cells could be disaggregated by mechanisms analogous to those outlined in the next section. The invasion by tumour cells of normal tissues in vivo is complicated by several factors, in particular infiltration of bloodborne mononuclear and sometimes polymorphonuclear cells. Hence the inflammatory changes which are seen, including oedema and tissue liquefaction, might arise from products of the tumour cells or the infiltrating cells, which have a high content of lysosomal hydrolases. However, when tumours invade cartilage there is little or no mononuclear infiltration, and yet breakdown of cartilage matrix is observed (see Poole, 1973). Electron microscopic studies have shown collagen degradation in basement membranes ahead of infiltrating ascites tumour cells (Birbeck and Wheatley, 1965) and skin and mammary tumour cells (Tarin, 1969). Hence it is probable that tumour cells are able to release enzymes that can degrade the proteoglycan matrix as well as the collagen fibres of connective tissues, including those in basement membranes. Further evidence that leucocytes are not required for tumour cell invasiveness comes from observations that virus-transformed BHK 21 cells can invade chick embryo organs and kidney explants in culture (Yarnell and Ambrose, 1969; Latner, Longstaff, and Pradhan, 1973).

The concept that tumour cells can liberate proteolytic enzymes, and that these may contribute to invasiveness, has long been held (Carrell and Ebeling, 1928). Various authors have shown that fibrin clots and denatured proteins are lysed in the presence of tumour cells. Among the proteases present in tumours are cathepsins B and D and dipeptidase (Sylvén, 1968a) and cathepsin B1 (assayed against benzylarginine naphthylamide by Blackwood et al, 1965). Several groups of investigators (Taylor, Levy, and Simpson, 1970; Dresden, Heilman, and Schmidt, 1972; Yamanishi, Dabbous, and Hashimoto, 1972) have reported the presence of collagenase in tumour cells, and epithelioma and 
sarcoma cells can digest a layer of reconstituted collagen on which they have grown. Since cathepsin B1 has collagenolytic activity (Burleigh, 1973) its presence in tumours may account for at least some of the activity observed.

The role of lysosomal enzyme release from intact cells in degradation of cartilage proteoglycan matrix has been demonstrated by Dingle, Barrett, and Weston (1971). The use of specific antibodies against the different proteases is clarifying their role in this process.

The addition of trypsin was found by Yarnell and Ambrose (1969) to increase the ability of virustransformed BHK 21 cells to invade chick embryo organs in culture. Latner et al (1973) reported that the protease inhibitor Trasylol decreased the invasion of mouse kidney explants by virus-transformed BHK 21 cells. These experiments are consistent with the interpretation that proteolytic enzymes contribute to the invasion of normal tissues by malignant cells. Studies with antibodies and other selective enzyme inhibitors should help to establish the role of different enzymes in this process.

The possibility that glycosidases also play a role in the breakdown of connective tissue matrix and spread of tumour cells must also be considered. Carr (1963) found that the growth of mouse tumours transplanted subcutaneously was inhibited by using separately or together inhibitors of beta-glucuronidase and beta- $\mathrm{N}$-acetylglucosaminidase (saccaro-1, 4-lactone, and 2-acetamido-2-deoxygluconolactone). The decreased tumour growth was attributed to inhibition of the degradation of hyaluronic acid and chondroitin sulphate, and hence the penetration of connective tissue matrix by the tumours. It would be of interest to compare the effects of such agents on tumour cell growth in vivo and in culture, since absence of effects in vitro would make improbable any direct effect on the growth of the tumour cells themselves.

\section{Proteases and Detachment of Tumour Cells}

Tumour cells usually adhere to one another relatively strongly, and the detachment of viable cells from the main tumour cell mass, with penetration into lymphatics or blood vessels, is required for metastatic spread. Since trypsin and other proteases disaggregate cells, the possibility that lysosomal proteases have similar effects has been examined. Sylvén (1968b) reported that a partially purified preparation of cathepsin B1 from calf liver caused detachment of BHK 21 hamster cells from glass at pH 7·1. Weiss (1962) found that addition of excess vitamin A facilitated detchment of cells from the glass on which they were cultured. Exposure of cells to high levels of vitamin A promotes secretion of lysosomal hydrolases into the surrounding medium. Weiss and Holyoke (1969) studied three groups of mice: one normal, another on a calorie-deficient diet, and a third treated with high doses of vitamin A. They found that if comparable spontaneous mammary tumours from the three groups were incubated at $37^{\circ} \mathrm{C}$, there was more release of tumour cells in the group treated with vitamin A than in the controls. When lysosomal homogenates were added, the cell release in all groups was considerably increased, the effect again being most marked in the group treated with vitamin A. It was also observed that the mice treated with vitamin A had a significantly greater number of lung metastases than untreated or undernourished mice. Control experiments showed that vitamin A treatment had no detectable effect on the trapping of tumour cells in the lungs or the growth of metastatic lesions. The raised incidence of metastases in the vitamin Atreated group was therefore attributed to the increased likelihood of cells escaping from primary tumours into the blood stream.

Weiss and Holyoke postulate that exposure to low levels of antibodies directed against the tumour cells may provoke a release of hydrolases from these cells, with increased danger of local and metastatic spread. This is one of several mechanisms by which antibodies might enhance tumour cell growth.

Polliack and Levij (1969) applied vitamin A alcohol topically to papillomas and carcinomas induced in the epithelium of the cheek pouch with 7,12-dimethylbenz(a)anthracene (DMBA). An increase in the size and invasiveness of the tumours was noticed.

Studies with inhibitors of hydrolase release or activity should help to clarify the role of these enzymes in metastatic spread, and might eventually come to control it. Conversely, it would be of interest to know whether drugs known to restrict metastasis, such as ICRF 159(Le Serveand Hellmann, 1972), have any effect on the release of hydrolases from tumour cells.

\section{Autophagy in Hormone-dependent Regression}

As discussed further by Wyllie at this meeting (see p. 35), cell lysis can be induced by two main groups of mechanisms, one acting initially on the plasma membrane, and often involving extracellular agents (antibody with complement, sensitized lymphocytes, membrane cytotoxins, etc), whereas the other group acts primarily within cells. Since I think that the terms 'coagulative necrosis' and 'apoptosis' are cumbersome, I prefer to call these exocidal and endocidal processes ('killing from without' and 
'killing from within'). Exocidal processes are characterized by early entry of sodium ions into the cells (a measure of failure of integrity of the plasma membrane) and loss of viability. Endocidal processes can bring about marked structural changes within cells while maintaining the normal ionic distribution across the plasma membrane, so that viability is maintained for a remarkably long time.

The various types of killing of tumour cells by immune mechanisms provide examples of exocidal processes, and the regression of hormone-dependent mammary tumours in rats following withdrawal of the required hormones illustrates an endocidal process. As pointed out by Gullino, Lanzerotti, and their colleagues (Gullino and Lanzerotti, 1972; Lanzerotti and Gullino, 1972), in this type of regression there is no impairment of blood flow or early infiltration of mononuclear cells. Gullino and Lanzerotti (1972) found that there is no change in the sodium space per unit weight of tumour during regression, and the process remains reversible for several days; small pieces of apparently degenerate tumour transplanted to a host with the appropriate hormonal background develop into tumours. The total activities of several lysosomal enzymes (acid phosphatase, arylsulphatase, beta-galactosidase, and beta-glucuronidase) increased significantly after 48 hours of tumour regression. There was a marked rise in the proportion of free (non-sedimentable) acid hydrolases during the later stages of regression. Synthesis de novo of beta-galactosidase and betaglucuronidase probably occurred during hormonedependent regression. Samples of interstitial fluid showed no demonstrable lysosomal hydrolases and no increase in protein and phosphorus content, but an increase in amino acid content. The authors conclude that cell lysis results from an autophagic process occurring within each cell which does not alter the pericellular environment or endanger the survival of neighbouring cells.

In fact, the simplest interpretation of hormone dependency appears to be that the hormone is required to maintain membrane integrity within the cell, so that the different cell organelles are kept apart. When the hormone is withdrawn some intracellular membranes are altered in such a way that fusion can take place, with consequent autophagy. For some time at least the plasma membrane retains normal function.

\section{Lysosomal Changes in Cancer Therapy}

Marked morphological changes in normal cells submitted to $x$-radiation have been reviewed by Allison (1969). Changes in the lysosomes of mouse mammary carcinoma cells following radiotherapy and chemotherapy with cyclophosphamide have been reported by Brandes and Anton (1966) and Brandes, Sloan, Anton, and Bloedorn (1967). Following both these treatments the concentration and size of lysosomes in treated tissues increased, and the reaction products of the histochemical test for acid phosphatase were detected in the cytoplasm and in intercellular spaces. It was concluded that the cyclophosphamide and ionizing radiation provoked the release and activation of lysosomal hydrolases and this preceded degradative changes in the tumour cells. These changes were increased by concurrent treatment with vitamin A. The presence of hydrolases in intercellular spaces suggests that one of the effects of chemotherapy or radiotherapy is extracellular discharge of lysosomes. The cells also contained autophagosomes, which suggests that membranes of lysosomes had been changed in such a way that their likelihood of fusing with other intracellular organelles or with the plasma membrane had been increased.

\section{Pinocytosis in Tumour Cells and its Possible Applica- tion in Chemotherapy}

The concept that rapidly growing tumours are 'nitrogen traps', ingesting plasma and other proteins to a greater extent than normal tissues, has some experimental support. Busch and Starbuck (1964), after injections of proteins labelled with radioactive isotopes, found that the specific activity of the protein within the tumour was higher than that in the liver and other normal tissues studied. Easty, Yarnell, and Andrews (1964) found that several tumour cell types, cultured in vitro, showed considerable uptake of fluorescein-labelled proteins. Ghose, Nairn, and Fothergill (1962) found in vivo a substantial amount of protein conjugate taken up by cells of ascites and solid tumours, although Easty (1964) has reported that uptake of fluorescent proteins into tumours in vivo frequently involves macrophages and stroma.

Fluorescence microscopy of normal and malignant cells can be used to show that macromolecules as well as many drugs become concentrated in the lysosomal system (Allison and Young, 1969). Indeed it appears that there are two main pathways followed by drugs within cells. Some drugs enter the smooth-membrane endoplasmic reticulum, where they are metabolized, whereas others for the most part enter the lysosomal system. The factors which determine which pathway a drug will take are not yet fully understood. Small, lipophilic, unchanged molecules can enter erythrocytes (Schanker, Nafpliotis, and Johnson, 1961) and, since these cells do not show endocytosis, are thought to penetrate through 
the plasma membrane. In nucleated cells such drugs could pass through the cytoplasm to the smoothmembrane endoplasmic reticulum and other organelles. Basic or acidic drugs can form macromolecular complexes with plasma proteins or other carriers and enter cells by pinocytosis. The small vesicles containing the drugs fuse with lysosomes. The macromolecular carriers are digested, leaving the drug which can remain bound within the lysosome or escape into the surrounding cytoplasm. Lysosomes contain a specific acidic glycolipid that can bind basic or lipophilic drugs.

When a drug enters a cell by diffusion, as a rule the intracellular concentration will be less than the extracellular. If the drug is associated with a carrier that can be taken into cancer cells by pinocytosis and the carrier is digestible, a selective concentration in lysosomes might be achieved. Two examples can be used to illustrate the principle. The bicyclic peptide amanitins (molecular weight about 1000) are the main toxins of the toadstool Amanita phalloides. The toxins become bound to and inhibit RNA polymerase II. When beta-aminitin is injected into animals, hepatocytes are damaged whereas sinusoidal cells appear unaffected even after the administration of high doses of the drug. Conjugation of beta-aminitin with albumin greatly increases the hepatotoxicity of beta-aminitin. The conjugate, like other serum proteins, is taken up by the sinusoidal cells of the liver and causes their necrosis, whilst hepatocytes are only damaged secondarily. Trouet, Deprez-de Campaneere, and de Duve (1972) have complexed the antibiotic daunorubicin with DNA; the complex has no bacteriostatic effect, but when the DNA is digested by lysosomal extracts activity is restored. Using fluorescence microscopy the cytoplasm of cells exposed to the free drug was seen to become rapidly and diffusely fluorescent. However, cells treated with the complex become slowly fluorescent in localized cytoplasmic particles and only later in the cytoplasm. This is consistent with uptake of a complex with quenched fluorescence, digestion in the lysosomal system, and liberation of the free fluorescent drug. The DNAdaunorubicin complex was taken up by tumour cells and was found in mice to be somewhat more effective in treatment of L1210 leukaemia than daunorubicin, mainly because of the greater toxicity of the latter.

Gregoriadis and Ryman (1972) have reported the entrapment of enzymes, including beta-fructofuranosidase, or ${ }^{131}$ I-albumin, in liposomes-concentric bilayers of lipids alternating with aqueous compartments. After intravenous injection the liposomes and entrapped materials are rapidly taken up by phagocytic cells of the liver and spleen, and are recoverable from the lysosome fraction, in which lysosomal lipids are digested, releasing their contents. It seems that comparable uptake of material entrapped within liposomes into tumours might be achieved. The reticuloendothelial system might be protected in various ways, eg, by injection of the liposomes into the artery supplying the tumour, so that a high local concentration will be achieved whilst the liposomes are diluted in the general circulation.

\section{Proteases, Membrane Glycoproteins, and Growth Control}

Incubation of erythrocytes and tumour cells with trypsin or other proteases leads to cleavage of sialic acid-containing carbohydrate moieties from membrane glycoproteins. Since the negative surface charge of plasma membranes is largely due to sialic acid, such cleavage alters the surface charge of the cells (Cook, Heard, and Seaman, 1960). Often, but not always, tumour cells have a greater net negative charge than their normal counterparts (Forrester, Ambrose, and Stoker, 1964). Poste (1971) observed a decrease in surface coat thickness when cells were treated with excess vitamin $A$, and produced evidence that this was due to lysosomal enzyme digestion. Thus proteases can markedly affect membrane glycoproteins which may play a role in the control of growth and metabolism.

The addition of low concentrations of trypsin to confluent cultures of normal chick embryo cells releases them from density-dependent inhibition of mitosis (Sefton and Rubin, 1970). Contact inhibition of growth in nonmalignant fibroblasts was likewise reversed by treatment with amounts of trypsin too small to detach cells (Burger, 1970). Conversely, protease inhibitors such as beef pancreas trypsin inhibitor have been found to promote parallel alignment of hamster tumour cells in culture, to increase the adhesiveness of rounded cells, and to decrease cellular proliferation (Goetz, Weinstein, and Roberts, 1972).

Inhibitors of protease activity have been found to decrease the effectiveness of promotion by croton oil or phorbol ester of tumours induced in mouse skin by DMBA (Troll, Klassen, and Janoff, 1970). Treatment with the phorbol ester resulted in an increase in skin protease activities. Similar observations have been reported by Hozumi, Ogawa, Sugimuta, Takeuchi, and Umezawa (1972) with the protease inhibitor leupeptin.

The importance of membrane glycoproteins in control is illustrated by the mitogenic effects on lymphocytes of plant proteins (lectins) that combine selectively with their carbohydrate moieties. Phyto- 
haemagglutinin (PHA) and concanavalin A induce cell division in thymus-derived lymphocytes, whereas pokeweed mitogen exerts this effect on both types of lymphocytes (Greaves and Janossy, 1972). Although the initial effects of lectins are on plasma membrane glycoproteins, they induce endocytosis and lysosomal changes in lymphocytes, and Allison and Mallucci (1964) suggested that lysosomal products, perhaps proteases, might be involved in derepression of lymphocytes and other cells. Further evidence in support of this view has accumulated (Hirschhorn, Brittinger, Hirschhorn, and Weissmann, 1968), including the inhibition of PHA-induced lymphocyte transformation by cytochalasin B, which blocks endocytosis (Allison, 1971). Although it is not yet clear whether the effects of lectins and cytochalasin are exerted at the plasma membrane or some other site, the role of cell membrane glycoproteins in the control of cell growth, and the effects of proteases, clearly require further investigation.

\section{Summary}

Lysosomal enzyme activities are often higher in tumours than in the corresponding normal cells. This is related in part to the undifferentiated state of tumour cells. Relatively high proportions of lysosomal hydrolases not sedimentable with cytoplasmic particles are often found in tumours. This is related in part to the presence of autophagosomes, which are easily disrupted by homogenization, and to autolysis. Release of hydrolases from tumour cells may facilitate penetration of normal tissues and detachment from tumours of cells that can metastasize. Regression of hormone-dependent tumours is an endocidal event in which autophagosomal digestion plays an important part. Formation of autophagosomes and other lysosomal changes frequently occur during cancer theapy. Proteases, probably acting on membrane glycoproteins, can release cells from growth control.

\section{References}

Adams, R. L. P. (1963). Periodic activation of lysosomal enzymes during regeneration of the liver. Biochem. J., 87, 532-536.

Allison, A. C. (1969). Lysosomes and cancer. In Lysosomes in Biology and Pathology, edited by J. T. Dingle and H. B. Fell, vol. 2, pp. 178-204. North-Holland, Amsterdam.

Allison, A. C. (1971). Effect of cytochalasin on transformation of human peripheral blood lymphocytes by phytohemagglutinin. In Immunologic Intervention, edited by $\mathbf{J}$. W. Uhr and $\mathbf{M}$. Landy, p. 299. Academic Press, New York.

Allison, A. C., and Mallucci, L. (1964). Lysosomes in dividing cells, with special reference to lymphocytes, Lancet, 2, 1371-1373.

Allison, A. C., and Young, M. R. (1969). Vital staining and fluorescence microscopy of lysosomes. In Lysosomes in Biology and Pathology, edited by J. T. Dingle and H. B. Fell, vol.12, pp.601628. North-Holland, Amsterdam.

Bartholomew, E. M., Bartholomew, W. R., and Rose, N. R. (1969). Isoenzyme differences between a human diploid cell line, WI38 and SV-40-transformed WI-38. J. Immunol., 103, 787-794.

Birbeck, M. S. C., and Wheatley, D. N. (1965). An electron micro- scopic study of the invasion of ascites tumour cells into the abdominal wall. Cancer Res., 25, 490-497.

Blackwood, C. E., Mandl, I., and Long, M. E. (1965). Proteolytic enzymes and their inhibitors in human gynecological tumours. Amer. J. Obstet. Gynec., 91, 419-429.

Bosmann, H. B. (1969). Glycoprotein degradation. Glycosidases in fibroblasts transformed by oncogenic viruses. Exp. Cell Res., 54, 217-221.

Boyland, E., Wallace, D. M., and Williams, D. C. (1955). The activity of the enzymes sulphatase and $\beta$-glucuronidase in the urine, serum and bladder tissue. Brit. J. Cancer, 9, 62-79.

Brandes, D., and Anton, E. (1966). The role of lysosomes in cellular lytic processes. III. Electron histochemical changes in mammary tumours after treatment with cytoxan and vitamin A. Lab. Invest., 15, 987-1006.

Brandes, D., Sloan, K. W., Anton, E., and Bloedorn, F. (1967). The effect of $\mathrm{X}$-irradiation on the lysosomes of mouse mammary gland carcinomas. Cancer Res., 27, 731-746.

Burger, M. M. (1970). Proteolytic enzymes initiating cell division and escape from contact inhibition of growth. Nature (Lond.), 227, 170-171.

Burleigh, M. C. (1973). Action on collagen of cathepsins B1 and D. Biochem. Soc. Trans., 1, 380-381.

Busch, H., and Starbuck, W. C. (1964). Biochemistry of cancer. Ann. Rev. Biochem., 33, 519-570.

Carr, A. J. (1963). The effect of some glycosidase inhibitors on experimental tumours in the mouse. Nature (Lond.), 198, 11041105.

Carrell, A., and Ebeling, A. H. (1928). The fundamental properties of the fibroblast and the macrophage. III. The malignant fibroblast of sarcoma 10 of the Crocker Foundation. J. exp. Med., 48, 105-123.

Cohen, S. L., and Bittner, J. J. (1951). The effect of mammary tumours on the glucuronidase and esterase activities in a number of mouse strains. Cancer Res., 11, 723-726.

Cohn, Z. A., and Fedorko, M. E. (1969). The formation and fate of lysosomes. In Lysosomes in Biology and Pathology, edited by J. T. Dingle and H. B. Fell, vol. 1, pp. 43-63. North-Holland, Amsterdam.

Conchie, J., and Hay, A. J. (1963). Mammalian glycosidases. 4. The intracellular localization of $\beta$-galactosidase, $\alpha$-mannosidase, $\beta-\mathrm{N}$-acetylglucosaminidase and $\alpha$-L-fucosidase in mammalian tissues. Biochem. J., 87, 354-361.

Conchie, J., and Levvy, G. A. (1957). Comparison of different glycosidase activities in conditions of cancer. Brit. J. Cancer, 11, 487-493.

Cook, G. M. W., Heard, D. H., and Seaman, G. V. F. (1960). A sialomucopeptide liberated by trypsin from the human erythrocyte. Nature (Lond.), 188, 1011-1012.

Deckers-Passau, L., Maisin, J., and de Duve, C. (1957). The influence of azodyes on lysosomal enzymes in rat liver. Acta Unio. Contra Cancerum, 13, 822-836.

Dingle, J. T. (1969). The extracellular secretion of lysosomal enzymes. In Lysosomes in Biology and Pathology, edited by J. T. Dingle and H. B. Fell, vol. 2, pp. 421-436. North-Holland, Amsterdam.

Dingle, J. T., Barrett, A. J., and Weston, P. D. (1971). Cathepsin D. Characteristics of immunoinhibition and the confirmation of a role in cartilage breakdown. Biochem. J., 123, 1-13.

Dresden, M. H., Heilman, S. A., and Schmidt, J. D. (1972). Collagenolytic enzymes in human neoplasms. Cancer Res., 32, 993-996.

Dzialoszynski, L. M., Fröhlich, A., and Kroll, J. (1966). Cancer and arylsulphatase activity. Nature (Lond.), 212, 733.

Easty, G. C. (1964). The uptake of fluorescent labelled proteins by normal and tumour tissues in vivo. Brit. J. Cancer, 18, 368-377.

Easty, G. C., Yarnell, M. M., and Andrews, R. D. (1964). The uptake or proteins by normal and tumour cells in vitro. Brit. J. Cancer, 18, 354-367.

Fishman, W. H., and Anlyan, A. J. (1947). B-glucuronidase activity in human tissues: some correlations with processes of malignant growth and with the physiology of reproduction. Cancer Res., 7, 808-817.

Fiszer-Szafarz, B., and Gullino, P. M. (1970). Hyaluronidase activity of normal and neoplastic interstitial fluid. Proc. Soc. exp. Biol. (N.Y.), 133, 805-807.

Forrester, J. A., Ambrose, E. J., and Stoker, M. G. P. (1964). Microelectrophoresis of normal and transformed clones of hamster kidney fibroblasts. Nature (Lond.), 201, 945-946.

Franklin, T. J. (1962). The influence of age on the activities of some acid hydrolases in the rat liver and kidney. Biochem. J., 82, 118-122. 
Ghose, T., Nairn, R. C., and Fothergill, J. E. (1962). Uptake of proteins by malignant cells. Nature (Lond.), 196, 1108-1109.

Goetz, I. E., Weinstein, C., and Roberts, E. (1972). Effects of protease inhibitors on growth of hamster tumor cells in culture. Cancer Res., 32, 2469-2474.

Goldberg, D. M., and Pitts, J. F. (1966). Enzymes of the human cervix uteri. Comparison of nucleases and adenosine deaminase in malignant and non-malignant tissue samples. Brit.J. Cancer, 20, 729-742.

Greaves, M., and Janossy, G. (1972). Elicitation of selective T and B lymphocyte responses by cell surface binding ligands. Transplant. Rev., 4, 87-130.

Gregoriadis, G., and Ryman, B. E. (1972). Lysosomal localization of $\beta$-fructofuranosidase-containing liposomes injected into rats. Biochem. J., 129, 123-133.

Gullino, P. M., and Lanzerotti, R. H. (1972). Mammary tumour regression. II. Autophagy of neoplastic cells. J. nat. Cancer Inst., 49, 1349-1356.

Hirschhorn, R., Brittinger, G., Hirschhorn, K., and Weissmann, G. (1968). Studies on lysosomes. XII. Redistribution of acid hydrolases in human lymphocytes stimulated by phytohemagglutinin. J. Cell Biol., 37, 412-423.

Horvat, A., and Touster, O. (1967). Biochemical characterization of the lysosomes of Ehrlich ascites tumour cells. Biochim. biophys. Acta (Amst.), 148, 725-740.

Hozumi, M., Ogawa, M., Sugimura, T., Takeuchi, T., and Umezawa, H. (1972). Inhibition of tumorigenesis in mouse skin by leupeptin, a protease inhibitor from actinomycetes. Cancer Res., 32, 1725-1728.

Karunairatnam, M. C., Kerr, L. M. H., and Levvy, G. A. (1949). The glucuronide-synthesizing system in the mouse and its relationship to $\beta$-glucuronidase. Biochem. J., 45, 496-499.

Lanzerotti, R. H., and Gullino, P. M. (1972). Activities and quantities of lysosomal enzymes during mammary tumour regression. Cancer Res., 32, 2679-2685.

Latner, A. L., Longstaff, E., and Pradhan, K. (1973). Inhibition of malignant cell invasion in vitro by a proteinase inhibitor. Brit. J. Cancer, 27, 460-464.

Le Serve, A. W., and Hellmann, K. (1972). Metastases and the normalization of tumour blood vessels by ICRF 159: a new type of drug action. Brit. med. J., 1, 597-601.

Levvy, G. A. (1956). Glucuronide metabolism, with specific reference to the steroid hormones. Vitam. and Hormones, 14, 267-303.

Page, R., Davies, P., and Allison, A. C. (1972). The role of macrophages in periodontal disease. In Host Resistance to Commensal Bacteria. The Response to Dental Plaque, edited by I. T. McPhee, pp. 195-201. Churchill Livingstone, Edinburgh and London.

Paigen, K. (1959). Genetic influence on enzyme localization. $J$. Histochem. Cytochem., 7, 248-249.

Polliack, A., and Levij, I. S. (1969). The effect of topical vitamin A on papillomas and intraepithelial carcinomas induced in hamster cheek pouches with 9,10-dimethyl-1,2-benzanthracene. Cancer Res., 29, 327-332.

Poole, A. R. (1973). Tumour lysosomal enzymes and invasive growth. In Lysosomes in Biology and Pathology, edited by J. T. Dingle, vol. 3, p. 83. North-Holland, Amsterdam.

Poste, G. (1971). Tissue dissociation with proteolytic enzymes
Adsorption and activity of enzymes at the cell surface. Exp. Cell Res., 65, 359-367.

Roy, A. B. (1958). Comparative studies on the liver sulphatases. Biochem. J., 68, 519-528.

Schanker, L. S., Nafpliotis, P. A., and Johnson, J. M. (1961). Passage of organic bases into human red cells. J. Pharmacol. exp. Ther. $133,325-331$.

Sefton, B. M., and Rubin, H. (1970). Release from density-dependent growth inhibition by proteolytic enzymes. Nature (Lond.), 227, 843-845.

Shamberger, R. J., and Rudolph, G. (1967). Increase of lysosoma enzymes in skin cancers. Nature (Lond.), 213, 617-618.

Strauch, L. (1972). The role of collagenases in tumour invasion. In Tissue Interactions in Carcinogenesis, edited by D. Tarin, $p$ 399. Academic Press, London.

Sylvén B. (1968a). Lysosomal enzyme activity in the interstitial fluid of solid mouse tumour transplants. Europ. J. Cancer, 4, 463-474.

Sylvén, B. (1968b). Cellular detachment by purified lysosomal cathep$\sin$ B. Europ. J. Cancer, 4, 559-562.

Tarin, D. (1969). Fine structure of murine mammary tumours: the relationship between epithelium and connective tissues in neoplasms induced by various agents. Brit. J. Cancer, 23, 417 425

Taylor, A. C., Levy, B. M., and Simpson, J. W. (1970). Collagenolytic activity of sarcoma tissues in culture. Nature (Lond.), 228 366-367.

Troll, W., Klassen, A., and Janoff, A. (1970). Tumorigenesis in mouse skin: inhibition by synthetic inhibitors of proteases. Science, 169, 1211-1213.

Trouet, A , Deprez-de Campaneere, D., and de Duve, C. (1972). Chemotherapy through lysosomes with a DNA-daunorubicin C complex. Nature [new Biol.], 239, 110-112.

Wagner, R. L., and Roth, J. S. (1967). Some properties of $\beta-$ glucuronidase activity in normal rat liver and in serveal transplantable rat hepatomas. Cancer Res., 27, 2053-2059.

Watanabe, K., and Fishman, W. H. (1964). Changes in hydrolase enzymorphology of rat uterus and vagina after estrogens; naphthol AS substrates. J. Histochem. Cytochem., 12, 908-918,

Wattiaux, R., Wattiaux de Coninck, S., van Dijck, J. M., Dupal, M. F., and Morris, H. P. (1970). Subcellular particles in tumors. IV. Lysosomes in hepatoma HC and Morris hepatomas 7794A, 7794B, 5123A, 7316A, and 16. Europ. J. Cancer, $6,503-515$.

Watts, C., and Goldberg, D. M. (1969). New observations on $\beta$ glucuronidase in human cervical cancer. Europ. J. Cancer, 5, 465-473.

Weiss, L. (1962). Cell movement and cell surfaces: a working hypothesis. J. theoret. Biol., 2, 236-250.

Weiss, L., and Holyoke, E. D. (1969). Some effects of hypervitaminosis A on metastasis of spontaneous breast cancer in mice. $J$. nat Cancer Inst., 43, 1045-1054.

Yamanishi, Y., Dabbous, M. K., and Hashimoto, K. (1972). Effect of collagenolytic activity in basal cell epithelioma of the skin on reconstituted collagen and physical properties and kinetics of the crude enzyme. Cancer Res., 32, 2551-2560.

Yarnell, M. M., and Ambrose, E. J. (1969). Studies of tumour invasion in organ culture. II. Effects of enzyme treatment. Europ. J. Cancer, 5, 265-269. 